\title{
Flux density spectrum of the magnetar AXP J1810-197
}

\section{K. Lazaridis ${ }^{1 *+}$ A. Jessner ${ }^{1}$, M. Kramer ${ }^{2}$, B. W. Stappers ${ }^{3}$, A. G. Lyne ${ }^{2}$, C. A. Jordan ${ }^{2}$, M. Serylak ${ }^{3}$ and J. A. Zensus ${ }^{1}$}

${ }^{1}$ Max-Planck-Institut für Radioastronomie

Auf dem Hügel 69, 53121, Bonn, Germany

${ }^{2}$ University of Manchester, Jodrell Bank Observatory

Macclesfield, Cheshire, SK11 9DL, UK

${ }^{3}$ Stichting ASTRON

Postbus 2, 7990 AA, Dwingeloo, the Netherlands

E-mail:klazarid@mpifr-bonn .mpg • de, jessner@mpifr-bonn .mpg • de,

Michael.Kramer@manchester.ac.uk, stappers@astron.nl,

andrew. lyne@manchester.ac.uk, cajejb.man.ac.uk, serylak@astron.nl,

azensusempifr-bonn.mpg.de

As part of a simultaneous multi-telescope observing campaign, we performed multi-frequency observations from July 2006 until March 2007 at 2.64, 4.9, 8.4, 14.6 and $32 \mathrm{GHz}$, with the 100$\mathrm{m}$ Effelsberg radio telescope of the Max-Planck-Institut für Radioastronomie, in order to obtain flux density measurements and spectral features of the $5.5 \mathrm{sec}$ radio emitting magnetar AXP XTE J1810-197. This magnetar consists of a main pulse (MP) and an interpulse (IP). We present the flux density spectrum of the average profile and of the separate pulse components of this first radio-emitting transient anomalous X-ray pulsar. We observe a decrease of the flux density of the order of 10 from July to December and also one of the two main components disappearing. Although the spectrum is generally flat with an average value for the spectral index of $\mathbf{a}=\mathbf{- 0 . 2 6}$ $+/-\mathbf{0 . 0 8}$, we observe intense fluctuations of the latter with time. For that reason we finally make some measurements of modulation indices for individual pulses in order to investigate the true nature of these fluctuations.

Bursts, Pulses and Flickering: Wide-field monitoring of the dynamic radio sky June 12-15 2007 Kerastari, Tripolis, Greece

\footnotetext{
* Speaker.

$\dagger$ Member of the International Max Planck Research School (IMPRS) for Radio and Infrared Astronomy at the Universities of Bonn and Cologne.
} 


\section{Introduction}

Magnetars, as first discussed by Duncan \& Thompson (1992), are considered to be slowly rotating neutron stars with spinning periods of 5-12 seconds and a rapid spin-down. They seem to possess extremely strong magnetic fields, typically $>10^{14} \mathrm{G}$, the decay of which is believed to create the observable high X-ray and gamma-ray luminosities, often expressed in bursts.

The model of magnetars tends to fit into two previously described distinct classes of objects, the soft gamma-ray repeaters (SGRs) and the Anomalous X-ray pulsars (AXPs). These objects were considered to be radio quiet, however, the pulsed radio emission that has been detected from AXP J1810-197 (Camilo et al. 2006) changed this belief.

The AXP XTE J1810-197 was revealed by Ibrahim et al. (2004) and it was discovered accidentally on July 2003 by the Rossi X-ray Timing Explorer (RXTE). With a period of $5.54 \mathrm{~s}$ and a period derivative $\sim 1.15 \cdot 10^{-11} \mathrm{~s} . \mathrm{s}^{-1}$, a magnetic field of $\sim 2.6 \cdot 10^{14}$ Gauss is implied. Although the previous easily classified it as an AXP, the extreme variations in the X-ray flux also classifies it as the first transient AXP.

The source is time variable (Camilo et al. 2007) and the radio emission is now becoming weaker, just as fast as it is believed that it became strong. Especially from July to December 2006 an extreme decrease of the radio flux density by an order of magnitude was observed with the $100 \mathrm{~m}$ Effelsberg Radiotelescope.

In this paper we report on the results of multi-frequency observations conducted at the radio frequencies of 2.64, 4.9, 8.4, 14.6 and $32 \mathrm{GHz}$ from July 2006 to March 2007. We present the average flux density spectrum of AXP XTE J1810-197 and the calculated value of its spectral index. Also the short term variability of the latter that led us to the measurements of the modulation indices for the individual pulses and the intra-day variability of their fluxes. However, a more detailed analysis on the single pulses will be reported elsewhere.

\section{Observations}

Observations were made using the 100-m radiotelescope of the Max-Planck Institute for Radioastronomy (MPIfR) at Effelsberg, Germany, as part of a simultaneous multi-telescope observing campaign of the European Pulsar Network (EPN). In total 3 quasi-simultaneous multi-frequency sessions in July 2006 were made and 8 more from December 2006 to March 2007. For the latter sessions the new subreflector of the telescope was used. It was installed in October 2006, improving the sensitivity and resulting in flatter gain curves at all frequencies. As an example, the sensitivity improvement at $32 \mathrm{GHz}$ is $47 \%$. Details of the observing sessions are summarized in Table 1.

\section{Data Analysis \& Results}

The quasi-simultaneous multi-frequency type of our observations enabled us to compute a spectral index for the magnetar for each of the observing sessions.Also by measuring the flux of the single pulses we were able not only to investigate the inter-day variability of the AXPJ1810197, which is the main purpose of this paper, but also the modulation of individual pulses during each of our sessions. 
Table 1: Summary of observing sessions.

\begin{tabular}{|c|c|c|c|c|c|}
\hline Date & Session & MJD & $\begin{array}{l}\text { Frequency } \\
(\text { GHz })\end{array}$ & $\begin{array}{c}\text { BW } \\
(\mathrm{MHz})\end{array}$ & $\begin{array}{c}\alpha \\
\text { Main Pulse | Interpulse }\end{array}$ \\
\hline \multicolumn{6}{|c|}{ July } \\
\hline $22 / 07 / 06$ & 1 & 53939.3 & $\begin{array}{c}8.35 \\
14.60\end{array}$ & $\begin{array}{l}1000 \\
2000\end{array}$ & $-2.06 \pm 0.08 \mid-1.99 \pm 0.28$ \\
\hline 26/07/06 & 2 & 53943.3 & $\begin{array}{c}4.85 \\
8.35 \\
14.60\end{array}$ & $\begin{array}{c}500 \\
1000 \\
2000\end{array}$ & $-0.25 \pm 0.06 \mid-1.29 \pm 0.26$ \\
\hline 28/07/06 & 3 & 53945.3 & $\begin{array}{c}4.85 \\
8.35 \\
14.60\end{array}$ & $\begin{array}{c}500 \\
1000 \\
2000\end{array}$ & $-0.02 \pm 0.14 \mid+0.28 \pm 0.57$ \\
\hline \multicolumn{6}{|c|}{ December-March } \\
\hline 09/12/06 & 1 & 54079.0 & $\begin{array}{c}2.64 \\
4.85 \\
8.35 \\
14.60\end{array}$ & $\begin{array}{c}100 \\
500 \\
1000 \\
2000\end{array}$ & $-0.35 \pm 0.11$ \\
\hline $26 / 12 / 06$ & 2 & 54096.0 & $\begin{array}{c}2.64 \\
4.85 \\
8.35 \\
14.60\end{array}$ & $\begin{array}{c}100 \\
500 \\
1000 \\
2000\end{array}$ & $-0.67 \pm 0.17$ \\
\hline $04 / 02 / 07$ & 3 & 54135.8 & $\begin{array}{c}2.64 \\
4.85 \\
8.35 \\
14.60\end{array}$ & $\begin{array}{c}100 \\
500 \\
1000 \\
2000\end{array}$ & $-0.29 \pm 0.49$ \\
\hline 06/02/07 & 4 & 54137.8 & $\begin{array}{c}4.85 \\
32.00\end{array}$ & $\begin{array}{c}500 \\
2000\end{array}$ & $-0.18 \pm 0.25$ \\
\hline $12 / 02 / 07$ & 5 & 54143.7 & $\begin{array}{c}4.85 \\
14.60\end{array}$ & $\begin{array}{c}500 \\
2000\end{array}$ & $+0.11 \pm 0.15$ \\
\hline $17 / 02 / 07$ & 6 & 54148.8 & $\begin{array}{c}4.85 \\
8.35 \\
14.60 \\
32.00\end{array}$ & $\begin{array}{c}500 \\
1000 \\
2000 \\
2000\end{array}$ & $+0.06 \pm 0.13$ \\
\hline $18 / 02 / 07$ & 7 & 54149.8 & $\begin{array}{c}4.85 \\
8.35 \\
14.60 \\
32.00\end{array}$ & $\begin{array}{c}500 \\
1000 \\
2000 \\
2000\end{array}$ & $+0.03 \pm 0.08$ \\
\hline $26 / 03 / 07$ & 8 & 54185.6 & $\begin{array}{c}4.85 \\
14.60 \\
32.00\end{array}$ & $\begin{array}{c}500 \\
2000 \\
2000\end{array}$ & $-0.80 \pm 0.17$ \\
\hline
\end{tabular}




\subsection{Average Flux Spectrum \& Spectral Index}

The measurement of the spectral properties of radio emission has already been done for many normal pulsars (Maron et al. 2000), (Löhmer et al. to be published) and showed that the majority of pulsars can be described by a simple power law with average value of spectral index $a=-1.8+/-$ 0.2. For the AXP J1810-197 Camilo et al. (2006) reported that it has a flat spectrum of $S \propto v^{-0.5}$ and that its flux density and the phase of the profile are varying intensively with time. Until August its integrated profile consisted of two prevailing features, the interpulse (IP) and the main pulse (MP). In the second group of our observing sessions, due to the decrease of its average flux density, one of those features is missing and we cannot be sure which one it is. The phase seems to change abruptly even from day to day. This short time phase change is similar to what was reported also by Camilo et al. (2007). For that reason in this section we will present the results from our second group of sessions and in $\mathbf{3 . 2}$ the results from our first one. A detailed analysis of the magnetars timing is in preparation (Lyne et al.) and we expect it to clear out this strange behaviour of the magnetar

Our results can be seen in Table 1. The spectrum of the AXPJ1810-197 that we measure is extremely flat with the spectral index value being $a=-0.26+/-0.08$, as presented in Figure 1 . This value is consistent with the literature (Camilo et al. 2006) and as mentioned before it provides us the opportunity to observe the magnetar at very high frequencies even in this stage when it seems to fade away. In May 2007 we observed single pulses from the magnetar at $43 \mathrm{GHz}$ for the first time, making this only the fifth neutron star observed at $7 \mathrm{~mm}$ with Effelsberg (Kramer et al. 1997).

Finally we present the fluctuation of the spectral index with time and the inter-day variability of the average flux density for every frequency. It can be seen in Figure 1 that the AXP J1810197 although it possesses a flat spectrum it seems that the spectral index fluctuates a lot around its mean value. The flux density from day to day is also not stable and it varies differently for different frequencies. Although it is not very clear, one could detect some similarities in the variation of the high frequencies (14.6 and $32 \mathrm{GHz}$ ) and the same for the low ones $(2.64,4.85$ and $8.35 \mathrm{GHz})$.

\subsection{Spectrum \& Spectral Index of the Interpulse}

As mentioned in $\mathbf{3 . 1}$ in the three July sessions we managed to take the results that are presented also in Table 1 for the MP and the IP. One can easily distinguish the huge difference in the signal strength between the two groups of our observations. By using the same method as before we measured the average flux density spectrum and we calculated the spectral index for the MP and the IP(Fig 2). The spectrum is not as flat as before and we also observe great variability with time where the spectral index tends to reach flatter values day by day.

Again we cannot distinguish, by comparing the spectral index of the two components, which is the component that we observe from December to March, because their spectra characteristics and widths are highly variable, due to the changes of the main pulse morphology.

\subsection{Modulation Indices}

Calculations of the modulation index of normal pulsars have been done with Effelsberg data before by Kramer et al. (2003) and Serylak et al. (2007) and also on the pulse to pulse flux density 

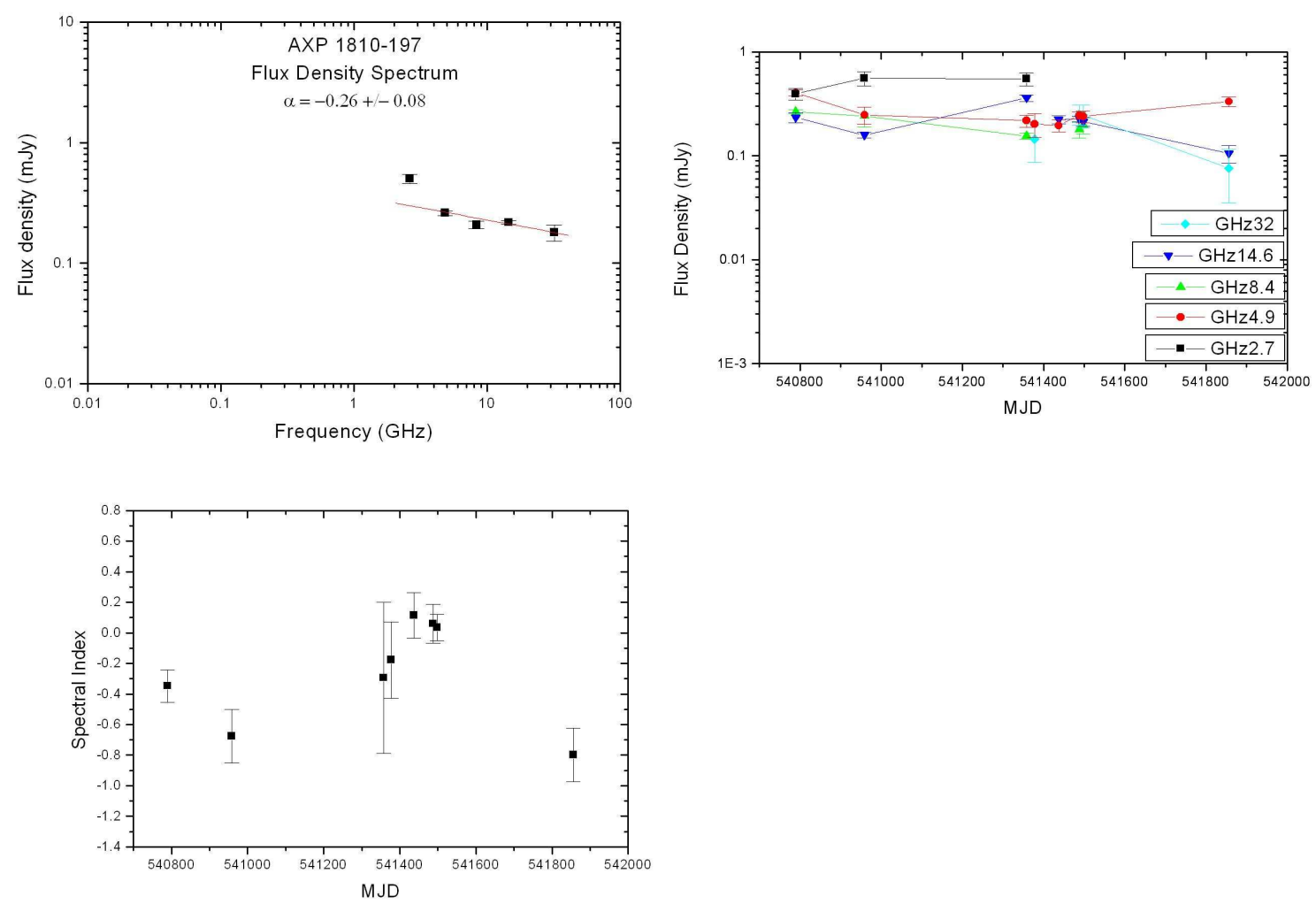

Figure 1: (Above Left) The flat mean flux density spectrum of the AXP J1810-197 with an average spectral index of $a=-0.26+/-0.08$. (Above Right) The inter-day variability of the AXP J1810-197. We see that the fluctuation is different for the different frequencies. (Below) The variability of the spectral index from day to day is great, however it stays (within $|a| \leq 1$ ) flat.
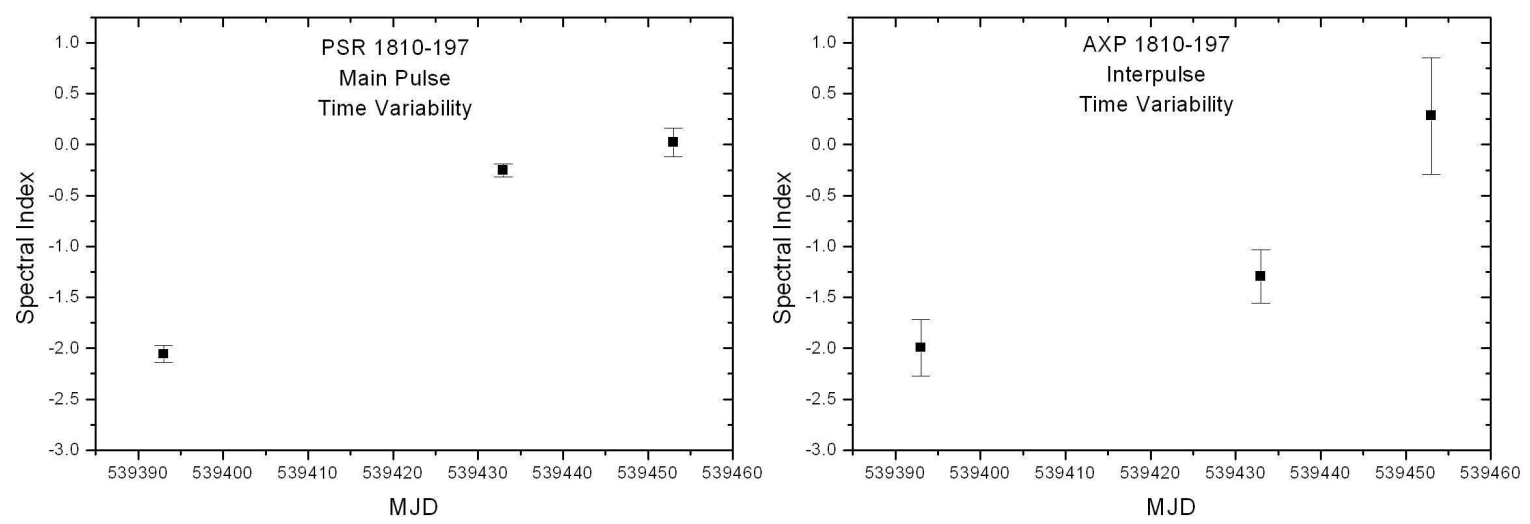

Figure 2: (Left) Variability of spectral index of MP with an average value of $a=-0.76+/-0.06$. (Right) Variability of spectral index of IP with an average value of $a=-1.00+/-0.23$. 

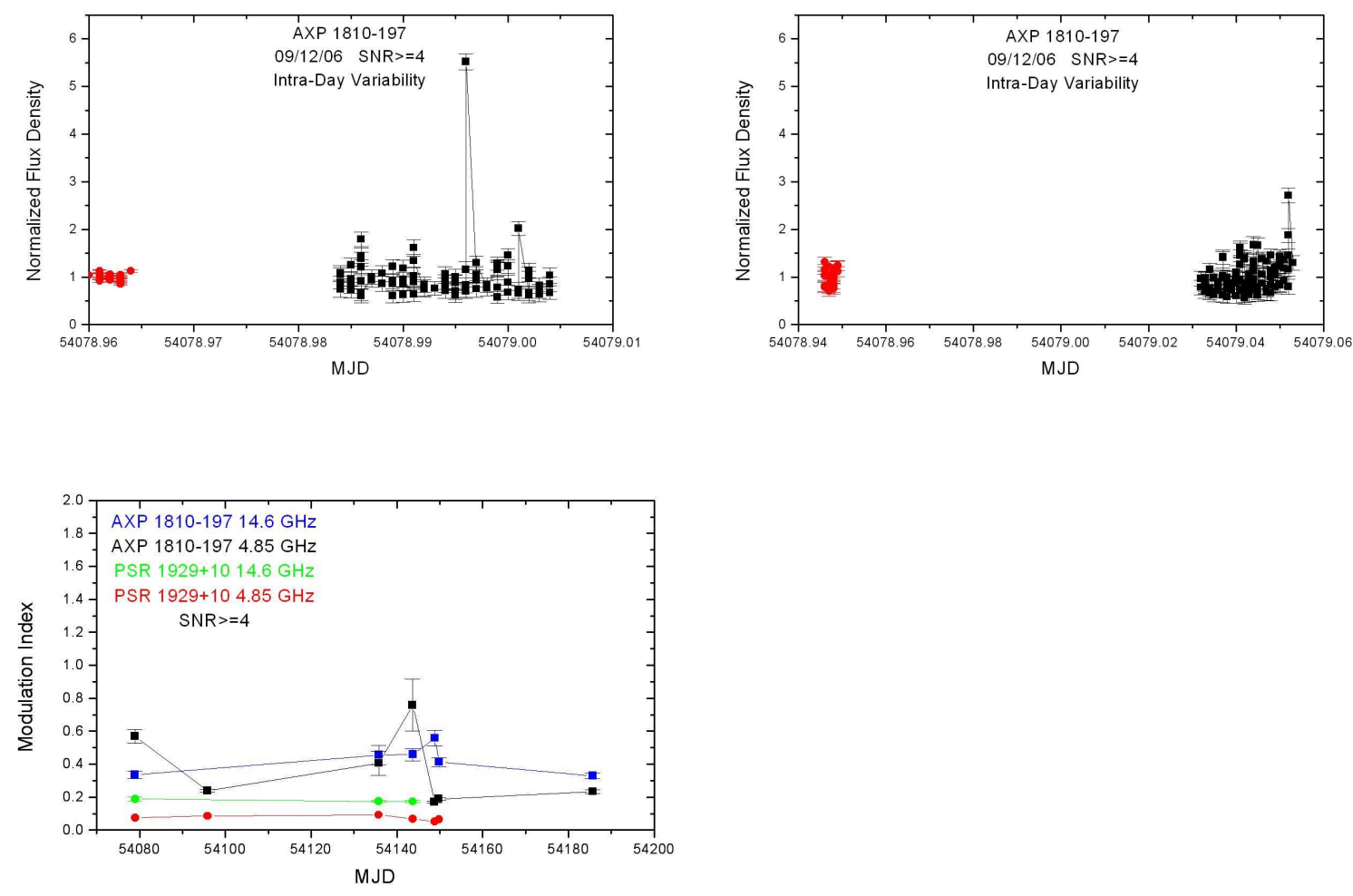

Figure 3: (Above Left) The intra-day variability of the normalized flux density of individual pulses for one day, at $4.85 \mathrm{GHz}$. (Above Right) The intra-day variability of the normalized flux density of individual pulses for one day, at 14.6 GHz. (Below) The inter-day variability of the modulation index of the AXP J1810-197 at 4.85 GHz (black line) and $14.6 \mathrm{GHz}$ (blue line). For comparison reasons and system stability checking the same for the PSR1929+10, at $4.85 \mathrm{GHz}$ (red line) and $14.6 \mathrm{GHz}$ (green line).

variability (Malofeev et al. 1995), with which detailed research in the intrinsic and interstellar modulation effects of pulsars was conducted.

Here we used the flux densities of individual pulses from our second group of observing sessions for two frequencies, $4.85 \mathrm{GHz}$ and $14.6 \mathrm{GHz}$. Because of the low flux signal we used only the single pulses when their flux density exceeded the signal-to-noise ratio (SNR) of 4 . So, all of our following results are under this limitation.

In Figure 3 we present the intra-day variability of the normalized flux density of single pulses of the AXP J1810-197 for a day at $4.85 \mathrm{GHz}$ and $14.6 \mathrm{GHz}$. With red are the points of our test pulsar PSR1929+10, which is a pulsar with well known properties that was observed before each one of the magnetars observations for comparison reasons. The main point is that we distinguish, by comparing with the test pulsar, that the intra-day fluctuation of the magnetar is also quite significant and that it is not due to system variability, in high and low frequencies. The final step of this paper is to present the modulation index $m$ as it varies with time. It is defined by $m^{2}=\frac{\left\langle(S-<S>)^{2}\right\rangle}{\langle S\rangle^{2}}$ (Kramer et al. 2003), where $S$ is the measured flux density and $\langle S\rangle$ its mean value. As can be seen in Figure 3 the modulation of the testpulsar is small and stable for both frequencies. This is not 
the case for the AXP J1810-197 though. Apart from the great variation that one could expect due to the previous results, there is also a difference in the way this happens for 4.85 and $14.6 \mathrm{GHz}$. Especially around day 54150 (12-18 February 2007) there is something that could be described as a mirroring of high and low frequencies.

\section{Summary \& Conclusion}

As mentioned, the quasi-simultaneous nature of our observations enabled us to regularly follow the magnetar's spectral behaviour, spectral variation and flux variation of the integrated profile and of the single pulses for almost 9 months. From the study of those observations the most definite conclusion one can have is that the observed behaviour of radio luminosity of AXP J1810-197 is not like any other pulsar we know.

First of all, it has flat and extremely variable average flux density spectrum, that allow us to observe it in high for other pulsars frequencies. Its spectrum seems to be described well with a single power law, which means that it does not show a turn-over, at least in the range of frequencies that we observed. Also, in the cases that we could see both, the spectrum of the MP and IP is quite different, being steeper for the IP and flatter for the MP. From that, one could suggest that during the low flux period of the magnetar (December-March) there might be times that we were actually observing the IP and times that we were seeing the MP or even different components of the MP, something that could be a convenient explanation for the extreme variation of the spectral index from day-to-day. However, the fact that even when we see both of the components the spectral variation from day to day is also significant, does not allow us to easily jump to these conclusions.

Thus, all the questions and answers seem to move around the variability of the magnetars emission. From what we observed we can definitely say that variability exists in all 3 time scales. The pulse-to-pulse modulation, the day-to-day variability and the long term of the order of months. Although the general trend is that at low frequencies the profile is becoming broader, which suggests interstellar scintillation, the variability of the modulation index does not seem to be connected with the pulse width at all, something that directly points to intrinsic variability during the day. Also we observe a kind of mirroring behaviour of the modulation index for the high and low frequencies from day-to-day. This variable behaviour definitely has to be examined more in detail in order to reveal the scintillation properties of the magnetar.

Because of the extremely variable behaviour of the magnetar, our observations are difficult to interpret in the frame of classical pulsar models. For that reason the more simultaneous or quasisimultaneous observing sessions from one or more telescopes we add to our analysis, the more information we get about the overall behaviour of it and the more we increase the chances to find trends and periodicities in the variable spectral properties.

\section{Acknowledgements}

Kosmas Lazaridis was supported for this research through a stipend from the International Max Planck Research School (IMPRS) for Radio and Infrared Astronomy at the Universities of Bonn and Cologne. Maciej Serylak was supported by the EU Framework 6 Marie Curie Early Stage Training programme under contract number MEST-CT-2005-19669 "ESTRELA". 


\section{References}

[1] F. Camilo, S. M. Ransom, J. P. Halpern, J. Reynolds, D. J. Helfand, N. Zimmerman, J. Sarkissian Transient pulsed radio emission from a magnetar, Nature, 442, 892C, (2006), [astro-ph/0605429].

[2] F. Camilo, I. Cognard, S. M. Ransom, J. P. Halpern, J. Reynolds, N. Zimmerman, E. V. Gotthelf, P. Demorest, G. Theureau, D. C. Backer, The Magnetar XTE J1810-197: Variations in Torque, Radio Flux Density, and Pulse Profile Morphology, ApJ, 663, 497C, (2007), [astro-ph / 0610685 ].

[3] F. Camilo, S. M. Ransom, J. Penalver, A. Karastergiou, M. Van Kerkwijk, M. Durant, J. P. Halpern, J. Reynolds, C. Thum, D. J. Helfand, N. Zimmerman, I. Cognard, The variable radio-to- $x$-ray spectrum of the magnetar XTE1810-197, submitted,(2007), astro-ph/0705.4095v1.

[4] R. C. Duncan, C. Thompson, Formation of very strongly magnetized neutron stars-Implications for gamma-ray bursts, ApJ, 392L, 9D, (1992).

[5] E. V. Gotthelf, J. P. Halpern, The spectral evolution of transient anomalous $x$-ray pulsar XTE J1810-197, ApJ, 632, 1075G, (2005), [asto-ph/0506511].

[6] E. V. Gotthelf, J. P. Halpern, The anatomy of a magnetar:XMM monitoring of the transient anomalous $x$-ray pulsar XTE J1810-197, Ap \& SS, 308, 79G, (2007), [astro-ph/ 0608473 ].

[7] J. P. Halpern, E. V. Gotthelf, R. H. Becker, Discovery of radio emission from the transient anomalous $x$-ray pulsar XTE J1810-197, ApJ, 632L, 29H, (2005), [astro-ph / 0508534 ].

[8] A. I. Ibrahim, C. B. Markwardt, J. H. Swank, S. Ransom, M. Roberts, V. Kaspi, M. P. Woods, S. Safi-Harb, S. Balman, W. C. Parke, C. Kouveliotou, K. Hurley, T. Cline, Discovery of a transient magnetar:XTE J1810-197, ApJ, 609L, 21I, (2005), [astro-ph / 0310665$].$

[9] M. Kramer, A. Jessner, O. Dorosenko, R. Wielebinski, Observations of pulsars at 7 millimeters, ApJ, 488, 364K, (1997).

[10] M. Kramer, A. Karastergiou, Y. Gupta, S. Johnston, Simultaneous single-pulse observations of radio pulsars. IV. Flux density spectra of individual pulses, $A \& A, 407,655 \mathrm{~K},(2003)$, [astro-ph/0306455].

[11] M. Kramer, B. W. Stappers, A. Jessner, A. G. Lyne, C. A. Jordan, Polarized radio emission from a magnetar, MNRAS, 377, 107K, (2007), [astro-ph/0702365].

[12] O. Löhmer, A. Jessner, M. Kramer, R. Wielebinski, O. Maron, Observations of pulsars at 9 millimetres, submitted, (2007).

[13] D. R. Lorimer, M. Kramer, Handbook of pulsar astronomy, Cambridge University press, Cambridge 2005.

[14] A. G. Lyne, F. Graham-Smith, Pulsar astronomy, Cambridge University press, Cambridge 2006.

[15] V. M. Malofeev, V. I. Shishov, W. Sieber, A. Jessner, M. Kramer, R. Wielebinski, Investigation of pulsar diffractive scintillation at 4.75 and 10.55 GHZ, A \& A, 308, 180M, (1996).

[16] R. N. Manchester, J. H. Taylor, Pulsars, Freeman, San Francisco 1977.

[17] M. Serylak, J. Kijak, O. Maron, A. Jessner, Pulse to pulse flux density modulation from pulsars at 8.35 GHz, submitted, (2007).

[18] P. M. Woods, C. Kouveliotou, F. Gavriil, V. M. Kaspi, M. S. E. Roberts, A. Ibrahim, C. B. Markwardt, J. H. Swank, M. H. Finger, X-ray bursts from the transient magnetar canditate XTE J1810-197, ApJ, 629, 985W, (2005), [astro-ph/0505039]. 